\title{
SF36 Quality of Life and Mortality across Different Levels of Serum Albumin in Patients with Hemodialysis
}

\author{
Tayebeh Soleymanian, ${ }^{1,}$ Maral Nejati, ${ }^{2}$ Mohsen Kabiri Esfahani, ${ }^{2}$ and Hassan Argani ${ }^{3}$ \\ ${ }^{1}$ Nephrology Research Center, Shariati Hospital, Tehran University of Medical Sciences, Tehran, IR Iran \\ ${ }^{2}$ Nephrology Research Center, Shariati Hospital, Tehran University of Medical Sciences-International Branch, Tehran, IR Iran \\ ${ }^{3}$ Urology and Nephrology Research Center, Modares Hospital, Shahid Beheshti University of Medical Sciences, Tehran, IR Iran \\ "Corresponding author: Tayebeh Soleymanian, Associate Professor of Nephrology, North Kargar Street, Shariati Hospital, Tehran University of Medical Sciences, Postal code: \\ 1411713135, Tehran, IR Iran. Tel: +98-2184902469, Fax: +98-2188026010, E-mail: soleymanian@tums.ac.ir
}

Received 2017 January 03; Accepted 2017 February 05.

\begin{abstract}
Background: Patients on hemodialysis (HD) generally display a significant decrease in the quality of life owing to comorbidities, malnutrition, and inflammation.

Methods: In this multicenter prospective study, the SF36 (short form with 36 questions scored between 0 and 100) and relevant demographic data and comorbidities (charlson comorbidity index); nutritional factors, and C-reactive protein (CRP) were evaluated in $416 \mathrm{HD}$ patients in September 2012. Hospitalization and mortality were assessed in a median of a 28 month follow-up.

Results: The SF36 score in survived patients was $53.6 \pm 19.3$ versus $41.6 \pm 22.4$ in the non-survived patients $(\mathrm{P}<0.001)$. There were significant adjusted inverse correlation between the SF36 score and age $(r=-0.14, P=0.005)$, diabetes $(r=-0.13, P=0.009)$, CCI $(r$ $=-0.24, P<0.001)$, serum CRP level $(r=-0.13, P=0.03)$, serum iPTH level $(r=-0.19, P<0.001)$, and also significant adjusted direct correlation with serum albumin $(r=0.15, P=0.003)$, and serum creatinine $(r=0.12, P=0.04)$. In Cox proportional hazard models adjusting for age, gender, dialysis vintage, diabetes and serum albumin, the hazard ratio of death for every 10 unit decrease in SF36 score was 1.10 (95\% CI: $1.04-1.22 ; \mathrm{P}=0.006)$. The adjusted hazard ratio for quintiles of serum albumin as $\leq 3.60,>3.60-3.85,>3.85$ $4.00,>4.00-4.20,>4.20$ (reference) g/dL was respectively 3.69 (1.98 - 6.89), 2.08 (1.10 - 3.94), 1.91 (0.97 - 3.85), 1.10 (0.76 - 1.49). Serum albumin revealed a strong association with mortality such that hazard ratio for every $1 \mathrm{~g} / \mathrm{dL}$ decrease in serum albumin was 6.28 (95\% CI: $3.80-10.42 ; \mathrm{P}<0.001$ ).
\end{abstract}

Conclusions: In patients with hemodialysis, SF36 shows significant association with serum albumin, comorbidities, inflammation, and clinical outcome.

Keywords: Hemodialysis, SF36 Quality of Life, Nutrition, Inflammation, Clinical Outcomes

\section{Background}

Health-related quality of life (HRQOL) appraises patient's well-being by the evaluation of both physical and mental scopes of health $(1,2)$. HRQOL of hemodialysis patients (HD) is threatened not only by the manifestations of the disease, but also by the burden of dialysis treatment. In addition, the quality of the communication between HD patients and health care providers or family members influences the HRQOL (3). It has been established that lower HRQOL is linked with higher risk of mortality and morbidity in hemodialysis patients (4-8). Therefore, the measurement of the HRQOL is a valuable strategy to assess high risk HD patients in order to implement a more effective ther$\operatorname{apy}(9)$.

Several markers such serum albumin, hemoglobin, age, dialysis duration, diabetes, and other comorbidities have been described as potential risks for lower HRQOL in hemodialysis patients $(5-7,10,11)$. However, the relationship between these markers and HRQOL may be as mark- ers, causes, or mutual ones.

In this study, we aimed to measure the SF36 quality of life of the hemodialysis patients and to assess the association between SF36 scores and the markers of nutrition and inflammation. We, also, evaluated whether SF36 and its components were independent predictors of clinical outcomes.

\section{Methods}

In this observational study, 532 maintenance hemodialysis patients from nine facilities were enrolled in September 2012. The enrolled facilities whose authorities and medical staff signed to collaborate were from three different regions: two facilities with 167 patients from north of Tehran, five facilities with 217 participants from the center of Tehran, and two facilities with 148 patients from the south of Tehran. All patients had to be at least 18 years old while having received outpatient hemodialysis at least for two weeks. Out of 532 patients, 416 subjects 
(95\% on dialysis for more than three months) agreed to complete the validated Persian translation of the SF36 questionnaire (12). In addition, a comprehensive questionnaire comprising all demographics, comorbidities (Charlson comorbidity index, CCI), and laboratory data was completed for all the patients in September 2012. At least the last two to three constitutive laboratory data at the start of the study were recorded in the questionnaire (their mean was used for analysis). We used Charlson comorbidity index (CCI) (13) to determine the comorbidities of the patients at the start of the study. Because all the patients had renal disease, we omitted its score from CCI. Then, patients were followed up until February 2015. During the follow-up period (median of 28 months; minimum of 0.5 and maximum of 30 months), causes of hospitalizations and exit from hemodialysis including death was recorded. The last follow-up time was the last visit or whenever patients left hemodialysis because of renal recovery, transfer to peritoneal dialysis (PD), or undergoing transplantation (one month after PD transfer or transplantation). Patients who transferred to a different facility were followed there. Data was extracted from the administrative forms and medical records by well-trained medical students in collaboration with dialysis unit staff (and relevant nephrologists if needed). The study was approved by the specialized review boards and ethics committees. Informed patient consent was obtained.

The SF36 comprised 36 questions 35 of which were used to make 8 multi-item subscales which were (1) physical functioning; (2) role-physical; (3) bodily pain; (4) general health; (5) vitality; (6) social functioning; (7) roleemotional; and (8) mental health (8). A score from 0 (lowest HRQOL) to 100 (highest HRQOL) was obtained for each. These subscales of SF36 were condensed into two components. The first five subscales constituted of physical component summary (PCS) and the last five were made up of mental component summary (MCS), so that the subscales of general health and vitality were used in both components. The scores of the total SF36 score and the two components were counted as averaging of the subscales. The Cronbach's $\alpha$ coefficient for each subscale was $>0.88$.

The SF36 questionnaires were explained to the patients or their relatives (in the case of patient's illiteracy) by welltrained medical students, then were filled out at home and returned to the dialysis unit. Of course, some patients desired to complete the questionnaire with the help of the students at the dialysis unit.

\subsection{Statistical Methods}

Demographic characteristics and laboratory data of the patients were summarized using the percentage of the total, means ( \pm standard deviation, SD), or medians (interquarter range) as appropriate. Mean values of the last two-three laboratory results for each patient were used in the analyses. Categorical variables were compared using Chi-Square or Fisher's Exact Tests, and continuous variables were compared using T Tests or Mann-Whitney U and ANOVA or Kruskal-Wallis as appropriate. We used Pearson's correlation and Spearman Rho coefficient $r$ for the analysis of the associations between SF36, PCS, MCS scores and other variables. Multivariate regression analysis was performed to assess adjusted correlations controlled for age, gender, dialysis vintage, serum albumin, and diabetes. Cox proportional hazard models were used for the hazard ratios (HR) of death while controlling for the aforementioned covariates. Follow-up time for each patient was the time of event (death) or censoring (recovery, peritoneal dialysis, transplantation, or the last visit), whichever developed first. Death in the first month of the transfer to PD or transplantation was included. The data analysis was performed using SPSS version 19 (SPSS Inc., Chicago, IL). Significant level was considered $\mathrm{P}<0.05$.

\section{Results}

Table 1 shows the demographic, clinical, and laboratory data of the whole patients, and also it has compared the characteristics of the survived and non-survived patients. The mean age of patients was $57 \pm 15$ years $56.5 \%$ (n $=235)$ of which were men and $42 \%(n=174)$ had diabetes. Other causes of ESRD were glomerular diseases $(n=63,15 \%)$, urologic problems $(n=42,10 \%)$, hereditary disorders $(n=$ $21,5 \%)$, hypertension and unknown causes $(n=111,27 \%)$, and other uncommon causes ( $n=5,1 \%$ ).

During the follow-up period (747 patient years) a total of 123 (30\%) death happened (16.5 per 100 patient years) (Table 1). The causes of death included cardiovascular disease (CVD) (37\%), other causes superimposed on CVD (11.5\%), infections superimposed on CVD (20.5\%), infections (4\%), malignancy (7.5\%), cachexia (5.5\%), and other causes (14\%). In the univariate analysis, non-survived patients compared to survived ones were significantly more diabetic with older age, had higher comorbidities, greater CRP level (more inflammation), lower serum albumin and creatinine levels (more malnutrition). The SF36 scores and their components and subscales, except mental health and social functioning subscales which had a trend, were significantly lower in the non-survived patients. The SF36 score in the survived patients was $53.6 \pm 19.3$ versus $41.6 \pm 22.4$ in the non-survived patients $(\mathrm{P}<0.001)$. Both PCS and MCS were lower in the non-survived patients compared to the survived ones $(54.5 \pm 20.4$ versus $42.6 \pm 23.2$, and $55.1 \pm 20.5$ 
Table 1. Characteristics of 416 Patients on Maintenance Hemodialysis According to Survival

\begin{tabular}{|c|c|c|c|c|}
\hline Characteristics & All Patients $(N=416)$ & Survived $(\mathrm{N}=\mathbf{2 9 3}, \mathbf{7 0} \%)$ & Non-Survived $(\mathrm{N}=\mathbf{1 2 3}, \mathbf{3 0} \%)$ & PValue \\
\hline \multicolumn{5}{|l|}{ Demographic data } \\
\hline Age, y & $57.2 \pm 15.4$ & $54.4 \pm 15.3$ & $63.9 \pm 13.5$ & $<0.001$ \\
\hline Sex (male/female), \% & 56.5 (male) & $74 / 66$ & $26 / 34$ & 0.10 \\
\hline Body mass index, $\mathrm{kg} / \mathrm{m}^{2}$ & $24.5 \pm 4.5$ & $24.4 \pm 3.9$ & $24.5 \pm 5.5$ & 0.84 \\
\hline Hemodialysis vintage, moa & $27(11-68)$ & $27(11-69)$ & $26(8-67)$ & 0.55 \\
\hline Diabetes, yes, \% & $174(42)$ & $103(35)$ & $71(58)$ & $<0.001$ \\
\hline Charlson comorbidity index ${ }^{\mathrm{a}}$ (except renal disease) & $2(0-3)$ & $1(0-2)$ & $4(3-5)$ & $<0.001$ \\
\hline \multicolumn{5}{|l|}{ Laboratory data (serum) } \\
\hline C-reactive protein, mg/La & $2.9(1.1-6.9)$ & $1.6(0.9-3.3)$ & $7.8(3.8-11.8)$ & $<0.001$ \\
\hline Albumin, g/dL & $3.91 \pm 0.36$ & $3.99 \pm 0.32$ & $3.70 \pm 0.38$ & $<0.001$ \\
\hline Hemoglobin, g/dL & $10.6 \pm 1.6$ & $10.6 \pm 1.6$ & $10.4 \pm 1.5$ & 0.20 \\
\hline Creatinine, $\mathrm{mg} / \mathrm{dL}$ & $8.6 \pm 2.7$ & $8.99 \pm 2.84$ & $7.72 \pm 2.31$ & $<0.001$ \\
\hline Pre-dialysis BUN, mg/dL & $55.5 \pm 13.2$ & $56.2 \pm 13.4$ & $53.9 \pm 12.7$ & 0.095 \\
\hline Cholesterol, mg/dL & $149 \pm 37$ & $149 \pm 35$ & $148 \pm 41$ & 0.68 \\
\hline Transferrin, mg/dL & $250 \pm 60$ & $250 \pm 61$ & $250 \pm 58$ & 0.93 \\
\hline Phosphorous, mg/dL & $5.4 \pm 1.3$ & $5.4 \pm 1.2$ & $5.3 \pm 1.3$ & 0.54 \\
\hline Intact PTH, pg/mLa & $341(165-586)$ & $351(192-570)$ & $302(143-658)$ & 0.48 \\
\hline Single-pool, Kt/V & $1.31 \pm 0.19$ & $1.31 \pm 0.19$ & $1.29 \pm 0.19$ & 0.43 \\
\hline SF36 score & $50.1 \pm 20.9$ & $53.6 \pm 19.3$ & $41.6 \pm 22.4$ & $<0.001$ \\
\hline Physical functioning & $47.5 \pm 30.9$ & $53.4 \pm 28.9$ & $33.1 \pm 30.7$ & $<0.001$ \\
\hline Role-physical & $48.6 \pm 31.1$ & $53.7 \pm 29.7$ & $37.2 \pm 31.1$ & $<0.001$ \\
\hline Bodily pain & $61.1 \pm 31.5$ & $63.3 \pm 30.2$ & $55.6 \pm 34.1$ & 0.03 \\
\hline General health & $49.7 \pm 23.1$ & $51.9 \pm 22.2$ & $44.3 \pm 24.2$ & 0.002 \\
\hline Vitality & $48.2 \pm 26.5$ & $50.3 \pm 25.4$ & $42.8 \pm 28.1$ & 0.008 \\
\hline Social functioning & $50.7 \pm 29.9$ & $52.4 \pm 29.5$ & $46.6 \pm 30.6$ & 0.07 \\
\hline Role-emotional & $56.1 \pm 35.7$ & $59.8 \pm 33.8$ & $47.1 \pm 38.6$ & 0.002 \\
\hline Mental health & $59.2 \pm 26.6$ & $60.6 \pm 24.8$ & $55.5 \pm 30.2$ & 0.1 \\
\hline Physical component summary (PCS) & $51.1 \pm 21.9$ & $54.5 \pm 20.4$ & $42.6 \pm 23.2$ & $<0.001$ \\
\hline Mental component summary (MCS) & $52.7 \pm 21.8$ & $55.1 \pm 20.5$ & $47.3 \pm 23.8$ & 0.002 \\
\hline Hospitalization (per patient-year) $^{\mathbf{a}}$ & $0.80(0.00-1.55)$ & $0.40(0.00-0.83)$ & $2.76(1.38-4.00)$ & $<0.001$ \\
\hline
\end{tabular}

${ }^{\mathrm{a}}$ Values are expressed as median and interquarter range.

versus $47.3 \pm 23.8$, respectively), even though the PCS was worse than MCS.

The case-mix adjusted correlation coefficients between the SF36 score as well as its two components and demographic-laboratory values is shown in Table 2 . There were statistically significant adjusted inverse correlation between the SF36 score and age $(\mathrm{r}=-0.14, \mathrm{P}=0.005)$, diabetes $(\mathrm{r}=-0.13, \mathrm{P}=0.009)$, CCI $(\mathrm{r}=-0.24, \mathrm{P}<0.001)$, serum CRP level $(r=-0.13, P=0.03)$, serum iPTH level $(r$ $=-0.19, \mathrm{P}<0.001)$, and also significant adjusted direct correlation with serum albumin $(\mathrm{r}=0.15, \mathrm{P}=0.003)$, and serum creatinine $(\mathrm{r}=0.12, \mathrm{P}=0.04)$. The results for PCS was almost similar to the SF36 score, however, only diabetes, comorbidities, serum albumin and iPTH had significant adjusted correlation with MCS. Although annual hospitalization frequency had significant inverse correlation with SF36 and its components, it became insignificant after case-mix adjustment. Serum hemoglobin, phosphorus, and $\mathrm{Kt} / \mathrm{V}$ did not show any significant correlation with the SF36 measurements. There was a trend toward less SF36 score in female patients mostly because of their lower PCS. We, also, observed significant lower PCS with longer dialysis duration.

The comparison of the demographic data, laboratory data, and clinical outcomes across quintiles of SF36 score are shown in Table 3.

Table 4 shows the hazard ratios of death for different variables (the mean of two-three measurements at the start of the study) using Cox proportional hazard models adjusting for age, gender, dialysis vintage, diabetes, and serum albumin. Older age, diabetes, lower serum albumin, higher comorbidities, CRP level, and iPTH were independent predictors of death. Adjusted hazard ratio of death for diabetic patients was 1.53 (95\% CI: $1.06-2.21$; P = 0.02) com- 
Table 2. Adjusted Correlation Coefficients for SF36, PCS, and MCS Versus Different Variables (Adjusted for Age, Gender, Dialysis Vintage, Serum Albumin, Diabetes)

\begin{tabular}{|c|c|c|c|}
\hline Characteristics & SF36 & $\begin{array}{l}\text { Physical Health } \\
\text { Dimension }\end{array}$ & $\begin{array}{l}\text { Mental Health } \\
\text { Dimension }\end{array}$ \\
\hline Age, $y$ & $-0.14(0.005)$ & $-0.13(0.01)$ & $-0.07(0.14)$ \\
\hline Sex (female) & $-0.09(0.05)$ & $-0.08(0.09)$ & $-0.04(0.42)$ \\
\hline Diabetes (yes) & $-0.13(0.009)$ & $-0.11(0.03)$ & $-0.11(0.02)$ \\
\hline $\begin{array}{l}\text { Hemodialysis vintage, } \\
\text { y }\end{array}$ & $-0.08(0.09)$ & $-0.10(0.04)$ & $-0.06(0.20)$ \\
\hline $\begin{array}{l}\text { Annual hospitalization } \\
\text { frequency }\end{array}$ & $-0.07(0.16))$ & $-0.07(0.14)$ & $-0.07(0.18)$ \\
\hline $\begin{array}{l}\text { Charlson comorbidity } \\
\text { index(except renal } \\
\text { disease) }\end{array}$ & $-0.24(<0.001)$ & $-0.24(<0.001)$ & $-0.19(<0.001)$ \\
\hline $\begin{array}{l}\text { C-reactive protein, } \\
\mathrm{mg} / \mathrm{L}\end{array}$ & $-0.13(0.03)$ & $-0.17(0.008)$ & $-0.006(0.92)$ \\
\hline Serum albumin, $\mathrm{g} / \mathrm{dL}$ & $0.15(0.003)$ & $0.15(0.002)$ & $0.11(0.02)$ \\
\hline $\begin{array}{l}\text { Serum creatinine, } \\
\text { mg/dL }\end{array}$ & $0.12(0.04)$ & $0.11(0.05)$ & $0.10(0.10)$ \\
\hline Pre-dialysis BUN, mg/dL & $0.06(0.23)$ & $0.08(0.10)$ & $0.02(0.68)$ \\
\hline $\begin{array}{l}\text { Serum intact PTH, } \\
\text { pg/mL }\end{array}$ & $-0.19(<0.001)$ & $-0.22(<0.001)$ & $-0.18(0.001)$ \\
\hline $\begin{array}{l}\text { Body mass index, } \\
\mathrm{kg} / \mathrm{m}^{2}\end{array}$ & $-0.03(0.58)$ & $-0.5(0.35)$ & $0.007(0.89)$ \\
\hline $\begin{array}{l}\text { Serum Hemoglobin, } \\
\text { g/dL }\end{array}$ & $0.02(0.68)$ & $0.02(0.67)$ & $0.01(0.84)$ \\
\hline $\begin{array}{l}\text { Serum transferrin, } \\
\text { mg/dL }\end{array}$ & $0.06(0.25)$ & $0.05(0.38)$ & $0.05(0.33)$ \\
\hline $\begin{array}{l}\text { Serum phosphorous, } \\
\text { mg/dL }\end{array}$ & $0.007(0.88)$ & $0.007(0.89)$ & $0.03(0.53)$ \\
\hline Single-pool, Kt/V & $0.07(0.19)$ & $0.06(0.27)$ & $0.06(0.28)$ \\
\hline
\end{tabular}

pared to the non-diabetics. Hazard ratio for every 1 unit increase in CCI was 1.54 (95\% CI:1.42 - 1.66; P $<0.001$ ), and for every $1 \mathrm{mg} / \mathrm{L}$ increase in CRP was 1.09 (95\% CI:1.05-1.12; P < 0.001). The SF36 score revealed a strong association with mortality, such that the hazard ratio of death for every 10 unit decrease in the SF36 score was 1.10 (95\% CI: 1.04 - 1.22; $\mathrm{P}=0.006)$. Hazard ratio for every 10 unit decrease in PCS was 1.12 (95\% CI: $1.03-1.21 ; \mathrm{P}=0.009$ ), and there was a trend for more mortality with every 10 unit decrease in MCS (HR: 1.07, 95\% CI: 0.99 - 1.16; P=0.09). Patients older than 65 years had 1.58 (95\% CI: $1.10-2.28 ; \mathrm{P}=0.01$ ) higher hazard ratio of mortality. Serum albumin revealed a strong association with mortality such that hazard ratio for every $1 \mathrm{~g} / \mathrm{dL}$ decrease in serum albumin was 6.28 (95\% CI:3.80-10.42; $\mathrm{P}<$ 0.001). Furthermore, hazard ratio for the least quintile of SF36 score ( $\leq 31$ ) was 2.22 (95\% CI: 1.19 - 4.17; P = 0.01), and for the second quintile ( $>31-44)$ was 1.50 (95\% CI: 0.91-2.96; $\mathrm{P}=$ 0.09 ) using the highest quintile $(>70)$ as reference (Figure 1A). Hazard ratio of death for the least quintile of serum albumin $(\leq 3.60 \mathrm{~g} / \mathrm{dL}$ ) was 3.69 (95\% CI:1.98 - 6.89; P $<0.001$ ), for serum albumin $>3.60-3.85 \mathrm{~g} / \mathrm{dL}$ was 2.08 (95\% CI: 1.10 - 3.94; $\mathrm{P}=0.02)$, and for serum albumin $>3.85-4.00 \mathrm{~g} / \mathrm{dL}$ was 1.91 (95\% CI: $0.97-3.85 ; \mathrm{P}=0.06$ ) compared to the highest quintile of serum albumin ( $>4.20 \mathrm{~g} / \mathrm{dL}$ ) (Figure $1 \mathrm{~B}$ ).

Kaplan-Meier survival analysis of the patients based on
SF36 quintiles and serum albumin quintiles is shown in Figure $2 \mathrm{~A}$ and $2 \mathrm{~B}$.

\section{Discussion}

In this multicenter observational study, we showed that SF36 score including its components (mostly PCS and a trend for MCS) have strong and significant inverse association with the mortality of the patients with hemodialysis. This observation was persisted even after the adjustment for other confounders such as age, gender, dialysis duration, diabetes, and serum albumin level. Notably, Patients in the lowest quintile of SF36 had 2.2 folds higher adjusted risk of death when compared with those in the highest quintile. There was also significant inverse association between SF36 and annual hospitalization frequency, although it was mitigated after adjustment for foregoing covariates.

PCS was the most significant component of SF36 score that had negative impact on the outcome and the effect of MCS was marginal. Accordingly, most other studies have shown stronger impact of PCS on mortality and hospitalization compared to MCS $(4,6)$. Also, subscales of PCS and MCS, except mental health and social functioning subscales which revealed only a trend, were significantly linked to mortality. Consistent with other reports, we described that serum albumin was one of the strongest independent predictors of patient outcome $(5,6,14,15)$. In this study, we showed that the ideal serum albumin was higher than $4 \mathrm{~g} / \mathrm{dL}$, and the risk of death was respectively 3.7, 2.1, and 1.9 folds in patients with the serum albumin $\leq$ $3.60 \mathrm{~g} / \mathrm{dL}$ (the lowest quintile), $>3.60-3.85 \mathrm{~g} / \mathrm{dL}$, and $>3.85$ - $4.00 \mathrm{~g} / \mathrm{dL}$ compared to patients with serum albumin of more than $4.20 \mathrm{~g} / \mathrm{dL}$ (the highest quintile).

The most significant association was between SF36 and underlying comorbidities and serum albumin. The present study revealed that diabetes is associated with both lower PCS and MCS. The association between SF36 and PCS with CRP concentration was fairly powerful. Age independently influenced the quality of life and women had worse SF36 scores compared to men mainly because of lower PCS.

We were not able to show a significant correlation between the level of hemoglobin and SF36, MCS, and specifically PCS of HD patients. Interestingly, we observed strong inverse correlation between serum PTH and SF36 score, PCS, MCS; and these associations became more significant after full adjustment for other variables. This finding indicates that hyperparathyroidism strongly affects PCS of HD patients via invoking abnormalities in bones and pain leading to physical disability. Interestingly, hyperparathyroidism influenced MCS of these patients as well. 
Table 3. Comparison of the Patients' Characteristics Across SF36 Quintiles

\begin{tabular}{|c|c|c|c|c|c|c|}
\hline Characteristics & 1st $\leq 31$ & 2nd $>31-44$ & $3 r d>44-55$ & 4 th $>55-70$ & 5 th $>70$ & PValue \\
\hline \multicolumn{7}{|l|}{ Demographic data } \\
\hline Age, $y$ & $64.1 \pm 14.1$ & $55.9 \pm 14.5$ & $56.4 \pm 15.8$ & $56.1 \pm 15.9$ & $53.6 \pm 15.1$ & $<0.001$ \\
\hline Sex (female), \% & 23.3 & 23.3 & 20.6 & 17.8 & 15 & 0.07 \\
\hline Body mass index, $\mathrm{kg} / \mathrm{m}^{2}$ & $24.7 \pm 5.8$ & $24.2 \pm 4.1$ & $25.1 \pm 4.6$ & $24.2 \pm 3.6$ & $23.9 \pm 3.8$ & 0.43 \\
\hline Hemoialysis vintage, $\mathrm{mo}^{\mathrm{a}}$ & $33(12-74)$ & $51(11-81)$ & $24(12-58)$ & $22(8-55)$ & $23(10-60)$ & 0.10 \\
\hline Diabetes (yes), \% & 25.9 & 20.1 & 20.1 & 19 & 14.9 & 0.04 \\
\hline Charlson comorbidity index (except renal disease) ${ }^{\mathrm{a}}$ & $3(2 \cdot 4)$ & $2(0-3)$ & $2(0-3)$ & $1(0-3)$ & $1(0-2)$ & $<0.001$ \\
\hline \multicolumn{7}{|l|}{ Laboratory data (serum) } \\
\hline C-reactive protein, mg/La & $4.4(1.6-11.3)$ & $2.9(1.2-7.1)$ & $3.0(1.1-5.7)$ & $2.1(1.1-7.1)$ & $2.2(0.9-5.0)$ & 0.07 \\
\hline Albumin, $\mathrm{g} / \mathrm{dL}$ & $3.79 \pm 0.39$ & $3.86 \pm 0.34$ & $3.95 \pm 0.37$ & $3.96 \pm 0.37$ & $3.98 \pm 0.32$ & 0.005 \\
\hline Hemoglobin, g/dL & $10.4 \pm 1.7$ & $10.6 \pm 1.4$ & $10.6 \pm 1.5$ & $10.5 \pm 1.6$ & $10.7 \pm 1.6$ & 0.74 \\
\hline Creatinine, mg/dL & $7.61 \pm 2.34$ & $8.40 \pm 2.56$ & $8.70 \pm 2.81$ & $8.82 \pm 2.59$ & $9.46 \pm 3.12$ & 0.001 \\
\hline Pre-dialysis BUN, mg/dL & $53.7 \pm 12.4$ & $54.1 \pm 11.2$ & $55.1 \pm 13.2$ & $56.4 \pm 16.4$ & $57.8 \pm 12.3$ & 0.25 \\
\hline Cholesterol, mg/dL & $146 \pm 31$ & $147 \pm 37$ & $152 \pm 41$ & $150 \pm 43$ & $151 \pm 30$ & 0.55 \\
\hline Transferrin, mg/dL & $237 \pm 55$ & $253 \pm 62$ & $253 \pm 66$ & $252 \pm 46$ & $253 \pm 66$ & 0.39 \\
\hline Phosphorous mg/dL & $5.17 \pm 1.24$ & $5.59 \pm 1.40$ & $5.40 \pm 1.31$ & $5.29 \pm 1.05$ & $5.57 \pm 1.28$ & 0.14 \\
\hline Intact PTH, pg/mLa & $376(143-658)$ & $390(167-730)$ & $353(153-630)$ & $328(213-557)$ & $282(165-500)$ & 0.48 \\
\hline Single-pool Kt/V & $1.29 \pm 0.19$ & $1.31 \pm 0.18$ & $1.30 \pm 0.21$ & $1.30 \pm 0.17$ & $1.35 \pm 0.21$ & 0.28 \\
\hline \multicolumn{7}{|l|}{ Clinical outcome } \\
\hline Hospitalizationa (per patient-year) & $1.2(0.4-2.8)$ & $0.8(0.4-2)$ & $0.6(0.0-1.5)$ & $0.4(0.0-1.0)$ & $0.4(0.0-1.2)$ & $<0.001$ \\
\hline Death,\% & 34.4 & 21.3 & 16.4 & 17.2 & 10.7 & $<0.001$ \\
\hline
\end{tabular}

aalues are expressed as median and interquarter range.

Figure 1. Hazard Ratio of Death by Quintile of Scores for A,SF36 Health-Related Quality of Life and Serum Albumin; B, Adjusted for Age, Gender, Dialysis Vintage, Serum Albumin, Diabetes

A

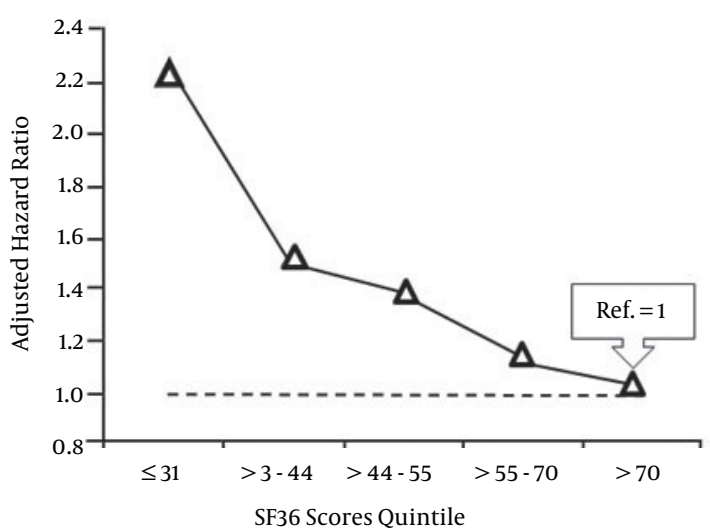

B

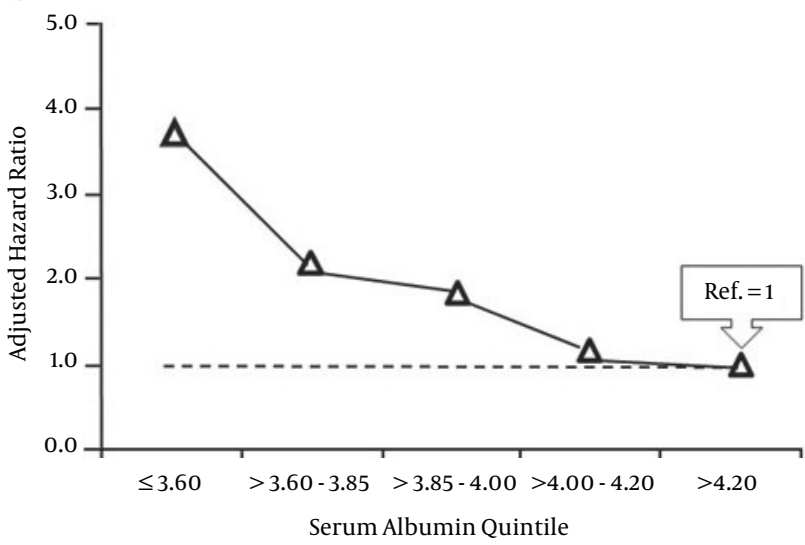

Hazard ratio and 95\% confidence interval for SF 36 score quintiles were respectively: $2.22(1.19-4.17 ; \mathrm{P}=0.01), 1.50(0.91-2.96 ; \mathrm{P}=0.09), 1.38$ (0.84 - 2.74), 1.16 (0.70 - 2.38); and for serum albumin quintiles these numbers were respectively: $3.69(1.98-6.89 ; \mathrm{P}<0.001)), 2.08(1.10-3.94 ; \mathrm{P}=0.02), 1.91(0.97-3.85, \mathrm{P}=0.06)$, and 1.10 (0.76 - 1.49).

In the present study, mental health subscale of patients did not significantly differ between survived and non-survived patients. Therefore, we speculated that religious beliefs, social support, and perhaps unawareness of the nature of their diseases should have some roles for this finding (16). The same result was seen for social functioning subscale which indicated non-survived patients had experienced a good social support of their family $(17,18)$.

Similar to most other studies we found that older age, diabetes, comorbidities, inflammation (higher CRP level), 
A

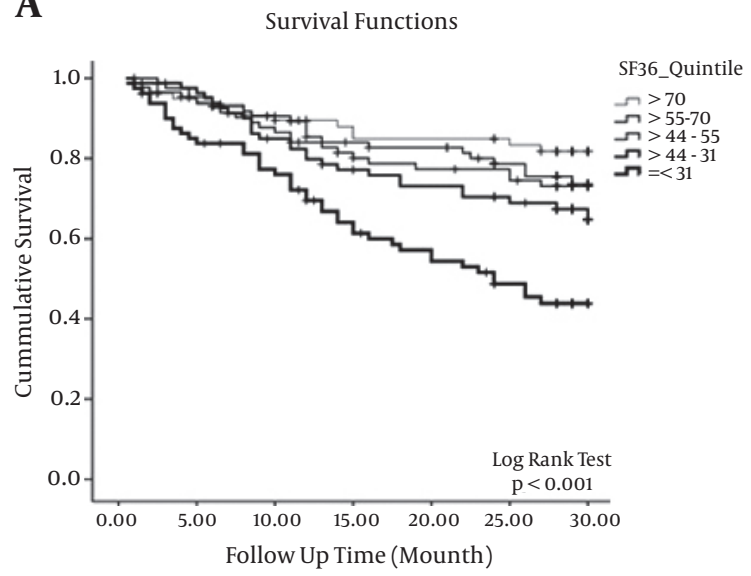

B

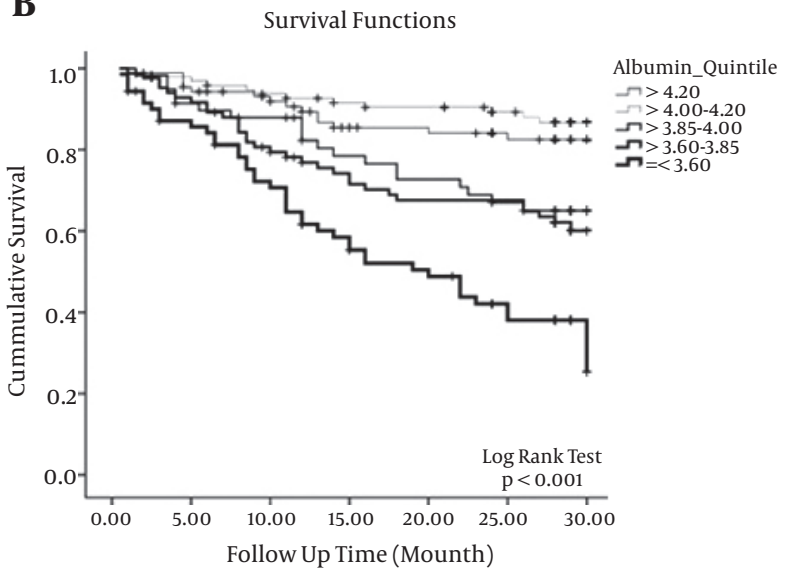

Figure 2. Kaplan-Meier Survival Plots for Different Quintiles of A, SF36 Score; B, serum albumin.

Table 4. Hazard Ratio of Death According to the Cox Proportional Hazards Model for SF36 and Selected Data (Adjusted for Age, Gender, Dialysis Vintage, Serum Albumin, Diabetes)

\begin{tabular}{lcc}
\hline Characteristics & $\begin{array}{c}\text { Hazard Ratio of Death (95\% } \\
\text { Confidence Interval) }\end{array}$ & P Value \\
\hline Age (every 1 year increase) & $1.02(1.01-1.04)$ & 0.001 \\
\hline Diabetes (yes) & $1.53(1.06-2.21)$ & 0.02 \\
\hline $\begin{array}{l}\text { Serum albumin (every 1 g/dL } \\
\text { decrease) }\end{array}$ & $6.28(3.80-10.42)$ & $<0.001$ \\
\hline $\begin{array}{l}\text { Serum C-reactive protein } \\
\text { (every 1 mg/L increase) }\end{array}$ & $1.09(1.05-1.12)$ & $<0.001$ \\
\hline $\begin{array}{l}\text { Serum iPTH, pg/mL (every } \\
\text { 100 pg/mL increase) }\end{array}$ & $1.08(1.02-1.14)$ & 0.006 \\
\hline $\begin{array}{l}\text { Charlson comorbidity index } \\
\text { (every one unit increase) } \\
\text { (except renal disease) }\end{array}$ & $1.54(1.42-1.66)$ & $<0.001$ \\
\hline $\begin{array}{l}\text { SF36 score (every 10 units } \\
\text { decrease) }\end{array}$ & $1.10(1.04-1.22)$ & 0.006 \\
\hline $\begin{array}{l}\text { PCS (every 10 units decrease) } \\
\text { MCS (every 10 units } \\
\text { decrease) }\end{array}$ & $1.12(1.03-1.21)$ & 0.009 \\
\hline
\end{tabular}

and malnutrition (lower serum albumin and creatinine levels) had negative impact on the quality of life (5-7, 10, $11,19,20)$. Some of these risk factors are modifiable and we have to implement strategies to improve nutrition and, also, fairly alleviate the inflammation and comorbid conditions. MCS was affected mostly by serum albumin, diabetes, and other comorbidities. Risk factors for lower PCS was the same as SF36 total score, however, PCS was influenced by dialysis vintage as well. Dialysis duration had no significant impact on the MCS of the patients denoting that they finely got along with their diseases.
In agreement with other observations, the present study showed that health-related quality of life measure (SF36 score) is a perfect, self-reported, and easy tool for the assessment of perceived physical and mental health of the patients with hemodialysis (5-7, 9). SF36 measure provides clues about the effects of various treatments on the quality of life. It also helps to recognize hemodialysis patients who are at a higher risk of morbidity and mortality in order to implement interventions to improve outcomes. However, it is not clear that the relationship between SF36 and outcomes is causal because most studies are observational and we need RCTs to conclude if interventions for promoting health-related quality of life also improve outcomes.

Limitations: SF36 score in this study was evaluated only once. Both SF36 score and serum albumin levels will be changed along the time course. However, for serum albumin we considered the mean of 3 constitutive results at the study start. Although SF36 score and particularly PCS had close relationship with clinical outcome, the causal effect of HRQOL on the outcome should be further determined by RCTs.

In summary, the present study provides a careful description of the HRQOL among HD patients and, also, determines important factors which are associated with a poor HRQOL. Malnutrition (lower serum albumin and creatinine), inflammation (higher CRP level), and comorbidities particularly diabetes are main risk factors for having worse HRQOL. Female and older patients suffer from additional physical health problems compared to men and younger counterparts. Intriguingly, hyperparathyroidism independently interferes with both physical and mental health components of HRQOL. Whereas dialysis duration can potentially affect PCS of dialysis patients, adaptation 
and coping with their diseases cause MCS less affected by dialysis vintage.

\section{Footnote}

\section{Conflict of Interest: No conflict of interest was declared.}

\section{References}

1. Curtin RB, Lowrie EG, DeOreo PB. Self-reported functional status: an important predictor of health outcomes among end-stage renal disease patients. Adv Ren Replace Ther. 1999;6(2):133-40. [PubMed: 10230880].

2. Kutner NG. Assessing End-Stage Renal Disease Patients' Functioning and Wellbeing: Measurement Approaches and Implications for Clinical Practice. Am J Kidney Dis. 1994;24(2):321-33. doi: 10.1016/s02726386(12)80198-3.

3. Valderrabano F, Jofre R, Lopez-Gomez JM. Quality of life in end-stage renal disease patients. Am J Kidney Dis. 2001;38(3):443-64. [PubMed: 11532675].

4. DeOreo PB. Hemodialysis patient-assessed functional health status predicts continued survival, hospitalization, and dialysis-attendance compliance. Am J Kidney Dis. 1997;30(2):204-12. [PubMed: 9261030].

5. Kalantar-Zadeh K, Kopple JD, Block G, Humphreys MH. Association among SF36 quality of life measures and nutrition, hospitalization and mortality in hemodialysis. JAm Soc Nephrol. 2001;12(12):2797-806. [PubMed: 11729250].

6. Mapes DL, Lopes AA, Satayathum S, McCullough KP, Goodkin DA, Locatelli $\mathrm{F}$, et al. Health-related quality of life as a predictor of mortality and hospitalization: the Dialysis Outcomes and Practice Patterns Study (DOPPS). Kidney Int. 2003;64(1):339-49. doi:10.1046/j.15231755.2003.00072.x. [PubMed: 12787427].

7. Md Yusop NB, Yoke Mun C, Shariff ZM, Beng Huat C. Factors associated with quality of life among hemodialysis patients in Malaysia. PLoS One. 2013;8(12):ee84152. doi: 10.1371/journal.pone.0084152. [PubMed 24358336].

8. Rostami Z, Einollahi B, Lessan-Pezeshki M, Soleimani Najaf Abadi A, Mohammadi Kebar S, Shahbazian H, et al. Health-related quality of life in hemodialysis patients: an Iranian multi-center study. Nephrourol Mon. 2013;5(4):901-12. doi: 10.5812/numonthly.12485. [PubMed: 24350090].
9. Hays RD, Kallich JD, Mapes DL, Coons SJ, Carter WB. Development of the kidney disease quality of life (KDQOL) instrument. Qual Life Res. 1994;3(5):329-38. [PubMed: 7841967].

10. Buckner S, Dwyer J. Do we need a nutrition-specific quality of life questionnaire for dialysis patients? J Ren Nutr. 2003;13(4):295-302. [PubMed: 14566767].

11. Anees M, Hameed F, Mumtaz A, Ibrahim M, Saeed Khan MN. Dialysisrelated factors affecting quality of life in patients on hemodialysis. Iran J Kidney Dis. 2011;5(1):9-14. [PubMed: 21189427].

12. Mehraban D, Naderi G, Salehi M. Development of SF-36 Questionnaire in the Measurement of Quality of Life in Patients on Renal Replacement Therapy in Iran. Saudi J Kidney Dis Transpl. 2003;14(1):15-7. [PubMed: 17657084].

13. Beddhu S, Bruns FJ, Saul M, Seddon P, Zeidel ML. A simple comorbidity scale predicts clinical outcomes and costs in dialysis patients. Am JMed. 2000;108(8):609-13. [PubMed:10856407].

14. Owen WJ, Lew NL, Liu Y, Lowrie EG, Lazarus JM. The urea reduction ratio and serum albumin concentration as predictors of mortality in patients undergoing hemodialysis. N Engl J Med. 1993;329(14):1001-6. doi: 10.1056/NEJM199309303291404. [PubMed: 8366899].

15. Woods JD, Port FK, Orzol S, Buoncristiani U, Young E, Wolfe RA, et al. Clinical and biochemical correlates of starting "daily" hemodialysis. Kidney Int. 1999;55(6):2467-76. doi: 10.1046/j.1523-1755.1999.00493.x. [PubMed: 10354296].

16. Kimmel PL, Emont SL, Newmann JM, Danko H, Moss AH. ESRD patient quality of life: symptoms, spiritual beliefs, psychosocial factors, and ethnicity. Am J Kidney Dis. 2003;42(4):713-21. [PubMed: 14520621].

17. Plantinga LC, Fink NE, Harrington-Levey R, Finkelstein FO, Hebah N, Powe NR, et al. Association of social support with outcomes in incident dialysis patients. Clin J Am Soc Nephrol. 2010;5(8):1480-8. doi: 10.2215/CJN.01240210. [PubMed: 20430940].

18. Tong A, Sainsbury P, Craig JC. Support interventions for caregivers of people with chronic kidney disease: a systematic review. Nephrol Dial Transplant. 2008;23(12):3960-5. doi:10.1093/ndt/gfn415. [PubMed: 18658178]

19. Germin-Petrovic D, Mesaros-Devcic I, Lesac A, Mandic M, Soldatic M, Vezmar D, et al. Health-related quality of life in the patients on maintenance hemodialysis: the analysis of demographic and clinical factors. Coll Antropol. 2011;35(3):687-93. [PubMed: 22053542].

20. Laws RA, Tapsell LC, Kelly J. Nutritional status and its relationship to quality of life in a sample of chronic hemodialysis patients.J Ren Nutr. 2000;10(3):139-47. [PubMed: 10921535]. 